\title{
Shiga toxin-producing Escherichia coli and their associated serotypes and virulence factors.
}

\section{Taís B. Vieira*, Taila S. Alves, Domingos S. Leite}

\begin{abstract}
Shiga toxin-producing Escherichia coli (STEC) is a group responsible for great part of gastrointestinal diseases in humans and for the high calf mortality rate. It is often associated with certain serotypes and other virulence factors. Here we tested STEC strains from feces collected from cohabitants farm animals to determine if they were serving as reservoirs of pathogenicity.
\end{abstract}

\section{Key words:}

Escherichia coli, colibacillosis, serotyping, virulence factors

\section{Introduction}

Shiga toxin-producing Escherichia coli (STEC) is a group responsible for great part of gastrointestinal diseases in humans and for the high calf mortality rate ${ }^{1}$. Healthy animals, especially bovines, might have STEC strains and be reservoirs that can later affect humans. STEC is often associated with a great variety of O:H serotypes, the most commonly related to human outbreaks being O157:H7. But when it comes to rough numbers, non0157 are the most prevalent serogroups ${ }^{2}$. In this study, Shiga toxin-producing $E$. coli strains were tested to determine their serogroups and other virulence factors that might be associated with the pathology brought by the Shiga toxin, such as adherence factors.

\section{Results and Discussion}

For this project, $675 \mathrm{E}$. coli strains were isolated from healthy animals' feces from two dairy farms (calf, cow, horse, mule, dog, lamb and sheep) and tested for Shiga toxin genes (stx1 and stx2) using PCR. A total of 108 $(16 \%)$ strains were positive for stx1 and/or stx2, all of them originated from calf, lamb or sheep. Antimicrobial susceptibility testing for those 108 strains showed 60 phenotypically distinct isolates, which were then tested against 31 different "O" antisera (Fig.1). Out of the 60 strains tested, 12 were positive for O22, 10 for 04,8 for O5, 4 for O91, 3 for O26, 2 for O103, 2 for O126, 1 for O2, 1 for O109, 1 for 0118 and 17 couldn't be serotyped (Table 1). The antimicrobial susceptibility testing (Fig.1) showed that five strains were classified as Multidrug Resistant (MDR, resistant to $\geq 3$ antimicrobial classes). All 108 strains were tested for $\mathrm{O} 157$ and $\mathrm{H} 7$ genes using PCR and none was positive. As for the associated virulence factors, 67 (62\%) strains were positive for the adherence factor genes (eae).

Figure 1. Methodology used in this study.



A: Disc diffusion method. B: Serotyping.
Table 1. Serogroups found and their respective strains.

\begin{tabular}{|c|c|}
\hline Serogroup & E. coli positive \\
\hline 02 & $B A 36 c$ \\
\hline 04 & $\begin{array}{l}\text { BA19c*; BA19d*; BA26b*; BB1a; BB1b; BB1c; }^{*} \text { BB1d; BB1e; CO1a; CO1e }\end{array}$ \\
\hline 05 & $\begin{array}{l}\text { BA24b; BA32a; BA36b; BA42c; BA44a*; } \\
\text { BA56e; BA57b; BA58c* }\end{array}$ \\
\hline 022 & $\begin{array}{l}\text { BA5a; BA5b; BA6a; BA6b; BA9a; BA9b; } \\
\text { BA16b; BA18a; BA18d; BA19c*; BA19d*; } \\
\text { BA26b* }\end{array}$ \\
\hline 026 & BA19c*; BA19d*; BA26b* \\
\hline 091 & BA44a*; BA58c*; CO1a; CN1d \\
\hline 0103 & BA30e; ВA36a \\
\hline 0109 & BA42b \\
\hline 0118 & BA33c \\
\hline 0126 & BA2a; BA3b \\
\hline
\end{tabular}

*Strains positive for more than one serogroup, needs further investigation. MDR isolate: ampicillin, amoxicillin + clavulanic acid, ciprofloxacin, enrofloxacin, chloramphenicol, tetracycline and sulfamethoxazole + trimethoprim.

\section{Conclusions}

Our findings indicate that the animals from which the isolates came from were serving as reservoirs for pathogenic $E$. coli, since they weren't sick themselves, but still carried pathogenic strains. This raises concerns about workers involved with the animals and about milk's contamination.

\section{Acknowledgement}

The authors thanks to FAPESP for the financial support (2015/15425-2; 2016/24885-0).

\footnotetext{
${ }^{1}$ Garcia, A., Ruiz-Santa-Quiteria, J.A., Orden, J.A. Prod. Animal. 1999; 147 19-372

${ }^{2}$ Blanco, M., Blanco, J.E., Mora, A., Dahbi, G., Alonso, M.P., González, E.A., Bernárdez, M.I., Blanco, J. J. Cli. Microbiol. 2004; 42(2): 645-651.
} 